\title{
Hippocampal Cytogenesis Correlates to Escitalopram-Mediated Recovery in a Chronic Mild Stress Rat Model of Depression
}

\author{
Magdalena N Jayatissa', Christina Bisgaard', Anders Tingström², Mariusz Papp ${ }^{3}$ and Ove Wiborg*,I \\ 'Centre for Basic Psychiatric Research, Aarhus Psychiatric University Hospital, Aarhus, Denmark; ${ }^{2}$ Molecular Psychiatry Unit, Wallenberg \\ Neuroscience Center, Lund, Sweden; 3 Institute of Pharmacology, Polish Academy of Science, Krakow, Poland
}

\begin{abstract}
From clinical studies it is known that recurrent depressive episodes associate with a reduced hippocampal volume. Conversely, preclinical studies have shown that chronic antidepressant treatment increases hippocampal neurogenesis. Consequently, it has been suggested that a deficit in hippocampal neurogenesis is implicated in the pathophysiology of depression. To study a potential correlation between recovery and hippocampal cytogenesis, we established the chronic mild stress (CMS) rat model of depression. When rats are subjected to CMS, several depressive symptoms develop, including the major symptom anhedonia. Rats were exposed to stress for 2 weeks and subsequently to stress in combination with antidepressant treatment for 4 consecutive weeks. The behavioral deficit measured in anhedonic animals is a reduced intake of a sucrose solution. Prior to perfusion animals were injected with bromodeoxyuridine (BrdU), a marker of proliferating cells. Brains were sectioned horizontally and newborn cells positive for BrdU were counted in the dentate gyrus and tracked in a dorsoventral direction.CMS significantly decreased sucrose consumption and cytogenesis in the ventral part of the hippocampal formation. During exposure to the antidepressant escitalopram, given as intraperitoneally dosages of either 5 or $10 \mathrm{mg} / \mathrm{kg} /$ day, animals distributed in a bimodal fashion into a group, which recovered (increase in sucrose consumption), and a subgroup, which refracted treatment (no increase in sucrose consumption). Chronic treatment with escitalopram reversed the CMS-induced decrease in cytogenesis in the dentate gyrus of the ventral hippocampal formation, but in recovered animals only. Our data show a correlation between recovery from anhedonia, as measured by cessation of behavioral deficits in the CMS model, and an increase in cytogenesis in the dentate gyrus of the ventral hippocampal formation.

Neuropsychopharmacology (2006) 3 I, 2395-2404. doi:I 0.1038/sj.npp. I 30 I04I; published online 8 February 2006
\end{abstract}

Keywords: chronic mild stress; depression; hippocampal formation; escitalopram; treatment resistance; cell proliferation; neurogenesis

\section{INTRODUCTION}

Back in the 1960s the monoamine hypothesis of depression was originally stated by Schildkraut and coworkers (Schildkraut, 1965) and has since then been in focus in numerous studies on antidepressant drug actions. The hypothesis is, however, incomplete and does not fully explain important clinical observations, like delay in onset of drug action and incomplete efficacy.

According to recent theories neuroplasticity, neurodegeneration and neurogenesis are key elements, underlying

\footnotetext{
*Correspondence: Dr $\bigcirc$ Wiborg, Centre for Basic Psychiatric Research, Aarhus Psychiatric University Hospital, Skovagervej 2, Risskov 8240, Denmark. Tel: + 457789 36। I, Fax: + 457789 3549,

E-mail: owiborg@post.tele.dk

Received 21 June 2005; revised 13 December 2005; accepted 20 December 2005

Online publication: 5 January 2006 at http://www.acnp.org/citations/ Npp010506050407/default.pdf
}

the pathology and pathophysiology of depression as well as antidepressant drug actions (Malberg and Schechter, 2005).

Stress is believed to be the most significant environmental factor in the etiology of depression, and neurons in the hippocampal formation are known to be hypersensitive to stress effects. The hippocampal formation undergoes morphological changes in response to stress, including atrophy and loss of CA3 pyramidal neurons (Magarinos et al, 1996), and stressful life events are known to suppress neurogenesis in the granule cell layer (GCL) of the hippocampal formation in different species (Gould et al, 1997, 1998; Tanapat et al, 1998). Accordingly, when an animal model for depression was used, a decrease in hippocampal volume was reported (Fuchs et al, 2004). Furthermore a reduced hippocampal volume was observed in depressed patients (Sheline et al, 2003; Bremner et al, 2000).

However, chronic treatment with different classes of antidepressants and electroconvulsive seizures is known to increase neurogenesis in the hippocampal formation of 
experimental animals (Santarelli et al, 2003; Madsen et al, 2000; Malberg et al, 2000). Furthermore, studies have demonstrated that chronic treatment with antidepressants reverses stress-induced inhibition of neurogenesis in the hippocampal formation (van der Hart et al, 2002; Malberg and Duman, 2003; Alonso et al, 2004; Fuchs et al, 2004; Czeh et al, 2001).

Chronic mild stress (CMS) in rodents has been proposed to model some of the environmental factors that contribute to the induction of depressive disorders in humans (Willner, 1997, 2005). Sequential exposure to a variety of mild stressors causes behavioral deficits in different paradigms that measure sensitivity to rewards. Thus, CMS suppresses the consumption of and preference for palatable sweet solutions (Willner et al, 1987), and the rewarding properties of food pellets, sweet solutions and amphetamine, as assessed by the place preference conditioning procedure (Papp et al, 1991). CMS furthermore increases thresholds for ventral tegmental self-stimulation, indicating a decreased responsiveness to rewarding stimuli (Moreau et al, 1995). This decrease in sensitivity to reward is assumed to model human anhedonia. Different classes of antidepressant medications have been shown to be effective in reversing the stress-induced decrease in sensitivity to reward. The drugs effective in the CMS model include tricyclic antidepressants (Papp et al, 1996), selective serotonin reuptake inhibitors (Sanchez et al, 2003), and monoamine oxidase inhibitors (Moreau et al, 1993). All antidepressants tested in the CMS model were effective at low to moderate dosages $(5-10 \mathrm{mg} / \mathrm{kg} / \mathrm{day})$, and a chronic drug exposure for 3-5 weeks was a prerequisite for obtaining full recovery.

Escitalopram is the active S-enantiomer of citalopram. In a chronic mild stress model of depression in rats, treatments with both escitalopram and citalopram were effective. Escitalopram showed a faster time of onset of action compared to citalopram and other antidepressants (Sanchez et al, 2003; Montgomery et al, 2001).

In the present study, we show that chronic mild stress causes a decrease in proliferation of new cells in the rat hippocampal formation and that this effect is reversed by chronic treatment with escitalopram. Furthermore, we found regional differences in sensitivity to stress and antidepressant actions in the hippocampal formation. Our main finding was, however, a correlation between the cell proliferation rate in the dentate gyrus and the anhedonic behavior in the CMS model for depression.

\section{MATERIALS AND METHODS}

\section{Animals}

Male Wistar rats were purchased from Taconic, Denmark. Animal weight was close to $200 \mathrm{~g}$ when adaptation for sucrose consumption was initiated, and approximately $350 \mathrm{~g}$ at the start of stress regime. The animals were singly housed, except when grouping was applied as a stress parameter. Food and water was available ad libitum except when food or/and water deprivation was applied as a stress parameter. The standard 12-h light/dark cycle was only changed in course of stress regime.

\section{Sucrose Consumption Test}

The animals were first trained to consume a palatable sucrose solution $(1.5 \%)$. The training lasted 5 weeks. In this period, the sucrose test was made twice a week during the first 3 weeks and once a week during the last 2 weeks. Animals were food and water deprived $18 \mathrm{~h}$ before the test. The test consisted of 1-h exposure to a bottle with sucrose solution. During the stress period the sucrose consumption test was performed once a week.

\section{Chronic Mild Stress Protocol}

On the basis of sucrose intakes in the three final baseline tests, the animals were divided into two matched groups and placed in separate rooms. One group was exposed to an initial 2 weeks of chronic mild stressors and the other was left undisturbed. The unchallenged group was food and water deprived $14 \mathrm{~h}$ before sucrose consumption test, otherwise food and water was freely available.

The stress procedure was optimized in our laboratory in Aarhus. The stress protocol was slightly modified from the protocol published by Papp (Sanchez et al, 2003). The stress protocol consisted of one period of intermittent illumination, stroboscopic light, grouping, food or water deprivation; two periods of soiled cage and no stress; three periods of $45^{\circ}$ box tilting. During grouping rats were housed in pairs with different partners alternately being a resident or intruder. All the stressors lasted from 10 to $14 \mathrm{~h}$. After the initial 2 weeks of exposure to stress, the unchallenged and stress groups were divided into two matched subgroups and subjected to chronic escitalopram or vehicle administration. Stress was continued during the entire period of treatment.

\section{Drug Administration}

Drug or vehicle was administrated intraperitoneally daily in the morning. Escitalopram (Lundbeck, Denmark) was dissolved in physiological saline and was given at two dosages of 5 and $10 \mathrm{mg} / \mathrm{kg}$.

\section{Bromodeoxyuridine (BrdU) Administration}

To label proliferating cells, the thymidine analogue 5-bromo2 -deoxyuridine was given to the rats intraperitoneally. Four injections of $\mathrm{BrdU}(100 \mathrm{mg} / \mathrm{kg}$ dissolved in phosphatebuffered saline (PBS) to a final concentration of $20 \mathrm{mg} / \mathrm{ml}$, $\mathrm{pH}=7.4$ ) were given at 2 -h intervals during one day. The animals were killed $16 \mathrm{~h}$ after the last injection.

\section{Tissue Processing}

After an anesthetic overdose (sodium pentobarbital $60 \mathrm{mg} /$ $\mathrm{ml}$ ) the animals were transcardially perfused with $100 \mathrm{ml}$ $0.9 \%$ saline followed by $200 \mathrm{ml}$ ice cold $4 \%$ paraformaldehyde $(\mathrm{pH}=7.2-7.4)$. The brains were then removed and postfixated overnight in the same solution at $4^{\circ} \mathrm{C}$. Brains were then transferred to a solution consisting of $30 \%$ sucrose (diluted in phosphate buffer, $\mathrm{pH}=7.0-7.4$ ) supplemented with $10 \%$ sodium azide and stored at $4{ }^{\circ} \mathrm{C}$ until they sank. The brains were sectioned horizontally along the entire dorsoventral axis $(-9.10$ to $-3.10 \mathrm{~mm}$ relative to 
bregma) (Paxinos and Watson, 1986) on a sliding microtome (HM 450, Microm). The $40 \mu \mathrm{m}$ sections were collected in series of every tenth (approximately 150 sections per brain) and stored in cryoprotectant antifreeze solution (25\% ethylene glycol and $25 \%$ glycerin in a 0.05 Í phosphate buffer) at $-20^{\circ} \mathrm{C}$.

\section{BrdU/NeuN Fluorescence Immunostaining}

The staining procedure was carried out on free-floating sections. The sections were rinsed $(3 \times 10 \mathrm{~min})$ in $0.02 \mathrm{M}$ potassium PBS (KPBS) and incubated in $1 \mathrm{M} \mathrm{HCL}$ for $30 \mathrm{~min}$ at $65^{\circ} \mathrm{C}$. Subsequently they were rinsed in blocking solution A ( $5 \%$ normal donkey serum NDS and 5\% normal horse serum NHS in $0.25 \%$ Triton-KPBS) for $1 \mathrm{~h}$ at room temperature (RT) and incubated with 1:100 anti-BrdU (Oxford Biotechnology, United Kingdom, OBT0030) and 1:100 anti-NeuN (Chemicon, CA, MAB377) in blocking solution $\mathrm{A}$ for $48 \mathrm{~h}$ at $4{ }^{\circ} \mathrm{C}$. After rinsing in $0.25 \%$ TritonKPBS $(2 \times 10 \mathrm{~min})$ and in blocking solution B $(2 \%$ NDS and $2 \%$ NHS in $0.25 \%$ Triton KPBS) $(1 \times 10 \mathrm{~min})$, the sections were incubated with 1:200 Cy3 donkey anti-rat (Jackson Immunoresearch, Pennsylvnia, Jackson 712-165-153) and with 1:200 biotinylated donkey anti-mouse (Jackson Immunoresearch, Pennsylvnia, Jackson 712-165-153) in blocking solution B for $2 \mathrm{~h}$ in darkness at RT. Sections were washed in $0.25 \%$ Triton-KPBS $(3 \times 10 \mathrm{~min})$ and incubated for $2 \mathrm{~h}$ with 1:200 Alexa-488-conjugated streptavidin (Molecular Probes, Oregon, S11223) in darkness at $\mathrm{RT}$. They were then rinsed in KPBS $(3 \times 10 \mathrm{~min})$ and stored in $\mathrm{KPBS}$ at $4^{\circ} \mathrm{C}$ and mounted on Superfrost Plus glass slides (Microm International, Randburg, Germany), airdried and coverslipped with a glycerol-based mounting medium.

\section{Data Quantification and Statistical Analysis}

Every 10th section throughout the entire hippocampal formation (the dorsal hippocampal formation -3.10 to $-4.28 \mathrm{~mm}$, the ventral hippocampal formation -4.60 to $-8.82 \mathrm{~mm}$ relative to bregma) (Paxinos and Watson, 1986) was analyzed by an observer unaware of the slide code. Sampling was carried out systematically and uniformly on random sections in the entire region of interest using conventional microscopy. For this purpose a Zeiss Axiovert $200 \mathrm{M}$ fluorescence microscope (Carl Zeiss International, Germany) with $\times 100$ objective (Plan-Neofluar $\times 100,1.30$ oil) was used. The BrdU-positive cells were counted in GCL including subgranular zone, hilus and molecular layer (ML) of the dentate gyrus. The total number of BrdU-labeled cells per dentate gyrus was estimated by multiplying the number of cells counted in every 10th section by 10 .

All data are presented as means \pm SEM. The sucrose data were analyzed using multivariate repeated measurement ANOVA (MANOVA) with stress $\times$ treatment as between subject factors and time as within subject factor. According to the statistically significant interactions revealed by MANOVA, separate analyses were performed to reveal specific differences between the groups. Following ANOVA analyses LSD post hoc tests were run.

The bimodal distribution of the stressed group treated with escitalopram (CMS-Es) was tested by a two component normal mixture with unequal variances against the null hypothesis that the data are best described by a single normal distribution (McLachlan et al, 2002). Testing was based on the likelihood ratio test statistic, the distribution of which was both (a) approximated as Chi-square with five degrees of freedom (Feng and McCulloch, 1996) and (b) bootstrapped using 1000 samples generated under the null. The weight of the rats was analyzed by ANOVA.

The difference in proliferating cell number between the groups was analyzed by ANOVA followed by LSD post hoc test. Statistical significance was set at $p<0.05$.

All procedures involving animals were accepted by Danish National Committee for Ethics in Animal Experimentation (2002/561-575).

\section{RESULTS}

\section{CMS Models Antidepressant Treatment Refraction}

After 2 weeks of initial exposure to chronic mild stress, the intake of sucrose solution was significantly diminished in the stress group compared to unchallenged animals, indicating a stress-induced decrease in sensitivity to reward (Figure 1). Chronic treatment with escitalopram $(5 \mathrm{mg} / \mathrm{kg} /$ day) reversed the decrease in sucrose intake in about $50 \%$ of the treated rats (responders). The remaining animals did not respond to the treatment (nonresponders).

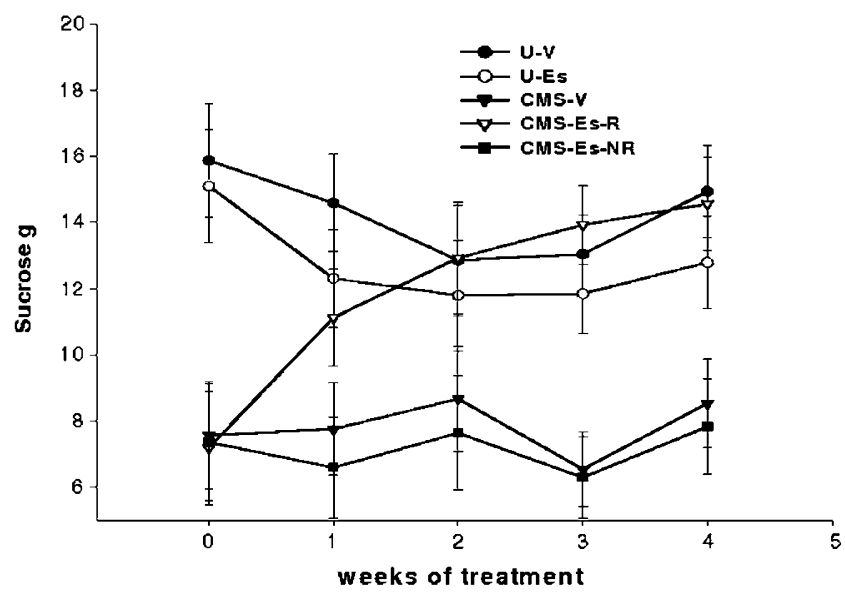

Figure I (a) Sucrose intake during 4 weeks of escitalopram treatment combined with continuous exposure to chronic mild stress (CMS). After 2 weeks of initial exposure to CMS, sucrose intake was significantly diminished in stress (CMS) compared to unchallenged $(U)$ animals (week $0)$. During 4 weeks of exposure to CMS the significant differences in sucrose consumption between the CMS-vehicle group (CMS-V) compared to the unchallenged-vehicle group $(U-V)$ were maintained $\left(F_{(5,12)}=6.15\right.$; $p=0.0047$ ).After I week of treatment the CMS-escitalopram group ( $5 \mathrm{mg}$ / $\mathrm{kg}$ ) split into two subgroups. According to the bimodal distribution animals were classified as either responders or nonresponders. Compared to the CMS-vehicle group (CMS-V) the sucrose intake increased in the responder (CMS-Es-R) $\left(F_{(4.13)}=4.7994 ; p=0.0134\right)$, but not in the nonresponder group (CMS-Es-NR) $\left(F_{(4,12)}=0.2277 ; p=0.917\right)$. The level of significance increased further in the course of time for the CMS-Es-R group. No significant differences were found in sucrose intake between the unchallenged escitalopram (U-Es) and unchallenged vehicle (U-V) group $\left(F_{(5,10)}=0.8745 ; p=0.5313\right)$. Both unchallenged groups were significantly different from the stress vehicle (CMS-V) group during all 4 weeks of treatment. Data are presented as means \pm SEM. 
We found the empirical density of the statistics describing the drinking behavior in the CMS-escitalopram group (CMS-ES) to be bimodal. A kernel density plot, which can be considered a refinement of a histogram or frequency plot, of the ratio of the average intake in week $1,2,3$, and 4, respectively, to the intake in week 0 , indicated a bimodal distribution (Figure 2). We investigated whether the observation of bimodality is indeed a result of the CMSES group consisting of two subpopulations, that is one responding to escitalopram, and one not responding. For the ratio of the average sucrose intake in weeks 1-4 over the intake in week 0, the null hypothesis that the data are best described by a single normal distribution was rejected regardless of whether test $(a)(p=0.034)$ or $(b)(p=0.013)$ was used to obtain critical values (see statistics in Materials and methods section). For the statistic under observation, this suggests both responders and nonresponders to be present in the CMS-ES group. In conclusion, certain features in the data, are consistent with the hypothesis that the CMS-ES group comprises both responders and nonresponders.

To test if the nonresponding phenomenon in the CMS model is dose dependent, we made a parallel behavioral study with a higher dose of escitalopram $(10 \mathrm{mg} / \mathrm{kg} /$ day $)$. The fraction of responders and nonresponders to drug treatment was highly comparable in both trials, i.e. a higher dose did not affect the number of nonresponders (Table 1).

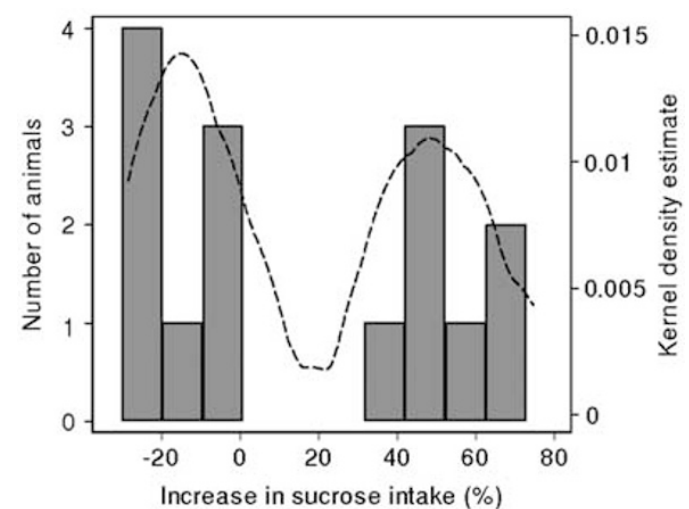

Figure 2 Bimodal segregation of escitalopram treated CMS animals into two subgroups of responders and nonresponders. The Kernel density plot, which is a frequency plot or refinement of a histogram, shows the bimodality in the frequencies of the relative increases in sucrose intake as average values for all 4 weeks of escitalopram treatment compared to baseline values.
Escitalopram treatment did not have any influence on sucrose drinking in chronically treated unchallenged animals. An insignificant fall in sucrose drinking in both unchallenged groups was probably caused by stress associated with daily i.p. injections. During the 4 weeks of treatment the intake of sucrose solution was constantly significantly higher in unchallenged compared to stress vehicle and stress escitalopram nonresponder groups (Figure 1).

No significant differences in body weight between the different CMS groups were found. The body weight was monitored at the initiation of the stress regime (unchallenged group: $325 \pm 5.1 \mathrm{~g}$ and CMS group $320.8 \pm 4.4 \mathrm{~g}$ ), at the initiation of drug treatment (unchallengedgroup: $383.9 \pm 7.5 \mathrm{~g}$ and CMSgroup $359.5 \pm 5.7 \mathrm{~g}$ ), and as end point measurements (unchallenged-escitalopram group: $398.5 \pm$ $13.4 \mathrm{~g}$, unchallenged-vehicle group: $401.6 \pm 17.1 \mathrm{~g}$, CMSvehicle group: $365.3 \pm 14.3 \mathrm{~g}$, responder-group: $368.5 \pm$ $10.1 \mathrm{~g}$, and nonresponder group $362.7 \pm 10.6 \mathrm{~g}$ ).

\section{Differential Regulation of Cytogenesis in the Hippocampal Formation}

In the present study, we used the CMS model to investigate the changes in cell proliferation rates in the dentate gyrus of the hippocampal formation of anhedonic rats as compared to rats who recovered from anhedonia after chronic treatment with escitalopram. New cells were identified by their ability to incorporate the DNA synthesis marker 5-bromo-2'-deoxyuridine (BrdU). To facilitate identification of the GCL and the subgranular zone (SGZ), mature neurons were stained with the neuronal marker NeuN.

When comparing unchallenged control animals with stressed animals, that is, group $\mathrm{U}-\mathrm{V} v s$ group CMS-V, we found a significant decrease $(42.2 \%)$ in the number of BrdU-positive cells in the GCL of CMS animals in the ventral part of the hippocampal formation, when estimating the number of new cells in dorsal and ventral part of the hippocampal formation separately (Figures 3 and 4). Importantly, the number of new cells was restored by escitalopram exposure in the responder group, whereas this effect was absent in the nonresponder group (Figures 3 and 4) The difference in the number of new cells between these two groups was statistically significant only in the ventral part of the hippocampal formation. In a plot of relative sucrose intake of all CMS animals treated with escitalopram $v s$ number of BrdU-labeled cells in the ventral hippocampal

Table I Number of Responders and Nonresponders after Chronic Escitalopram Treatment in the Chronic Mild Stress (CMS) Model

\begin{tabular}{lccccc}
\hline & \multicolumn{2}{c}{ Responders } & & Nonresponders \\
\cline { 2 - 5 } Escitalopram treatment & Number of responders & $\begin{array}{c}\text { Range of increase in } \\
\text { sucrose intake }\end{array}$ & & $\begin{array}{c}\text { Number of } \\
\text { nonresponders }\end{array}$ & $\begin{array}{c}\text { Range of increase in } \\
\text { sucrose intake }\end{array}$ \\
\hline $5 \mathrm{mg} / \mathrm{kg} /$ day & 8 & $55-340 \%$ & 7 & $0-10 \%$ \\
$10 \mathrm{mg} / \mathrm{kg} /$ day & 6 & $58-302 \%$ & & 7 & $0-7.3 \%$ \\
\hline
\end{tabular}

Two parallel behavioral trials revealed a dose-independent ratio of responders to nonresponders. The relative increase in sucrose consumption for nonresponders between weeks 0 and 4 was below $10 \%$. 


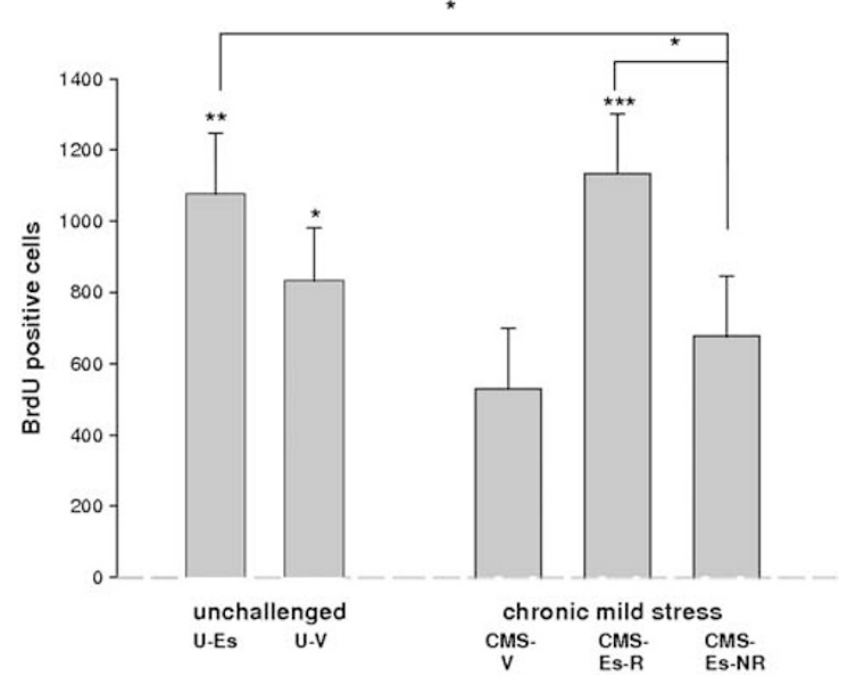

Figure 3 Total number of BrdU-positive cells in the granule cell layer (GCL) of the ventral hippocampal formation. Cytogenesis in GCL was significantly diminished by CMS (CMS-V) compared to the unchallenged groups (U-Es and $U-V)$. The stress-induced decrease was reversed by chronic escitalopram treatment $(5 \mathrm{mg} / \mathrm{kg}$ ) in the responder group (CMS-EsR) but not in the nonresponder group (CMS-Es-NR). The difference in number of new cells between responder and nonresponder groups was highly significant. No significant differences in the number of BrdU-positive cells were revealed between the unchallenged groups (U-Es and U-V). The number of new cells was significantly higher in the unchallenged escitalopram (U-Es) group compared to stress vehicle (CMS-V) and stress escitalopram nonresponder (CMS-Es-NR) groups. Data are presented as means \pm SEM and were analyzed with analysis of variance (ANOVA) and LSD post hoc test. Statistical significance was set at $p<0.05$. * $=p<0.05$, ** $=p<0.0 \mathrm{I}$, and $* * *=p<0.00 \mathrm{I}$ compared to stress vehicle group or as indicated.

formation we found an overall significant positive correlation $(p=0.036)$ (Figure 5).

We found no significant differences between the numbers of BrdU-labeled cells in the dentate hilus of the ventral hippocampal formation between the groups (Table 2). However, the general tendency of stress-related inhibition and normalization to control level after escitalopram treatment was sustained. In the ML of the ventral hippocampal formation, cell proliferation was significantly impaired in CMS compared to unchallenged animals. Chronic escitalopram treatment did not reverse this effect (Table 2).

A slightly lower, but insignificant, number of new cells in GCL were found in the dorsal part of the hippocampal formation in the stress vehicle group compared to the unchallenged vehicle group (Figure 6). However, in all animal groups treated with escitalopram, the number of BrdU-positive cells in GCL was significantly higher compared to stress vehicle animals (Figure 6). In contrast to the ventral part of the hippocampal formation, no differences in the number of BrdU-positive cells in GCL were found between the groups of responders and nonresponders in the dorsal hippocampal formation. An important observation concerning the dorsal hippocampal formation was that the number of BrdU-positive cells in GCL was significantly elevated $(111.8 \%)$ in unchallenged escitalopram-treated animals compared to the unchallenged vehicle group (Figure 6). No significant differences in cytogenesis in hilus in the dorsal hippocampal formation were found. In ML the cell proliferation was significantly elevated in unchallenged control group treated with escitalopram (data not shown).

\section{Regulation of Cytogenesis throughout the Entire Hippocampal Formation}

When estimating the number of progenitor cells in GCL of the entire hippocampal formation as a unit, the number of BrdU-positive cells was significantly higher in the responder group compared to the stress vehicle group, but no differences in ML and hilus were detected (Figure 7). Significantly higher BrdU-positive cell numbers in GCL, hilus, and ML were found in the unchallenged-escitalopram group compared with the CMS-vehicle group.

\section{DISCUSSION}

From a functional perspective the hippocampal formation, which is known to be involved in the pathophysiology of mood disorders including depression (Brown et al, 1999; Vaidya and Duman, 2001), is essentially regarded as a homogenous formation along the dorso-ventral axis. The focus is mainly on the different hippocampal subfields, dentate gyrus, CA1-CA4, and subiculum, which repeat themselves along the longitudinal axis of the hippocampal formation. However, when moving from the dorsal to the ventral part of the hippocampal formation, they have different efferent and afferent connectivities. Likewise, differences in constitution of the neuronal network are found within the dorsal and ventral regions (Moser and Moser, 1998). In the ventral hippocampal formation in rodents the density of dopaminergic, noradrenergic, and serotonergic terminals is higher (Gage and Thompson, 1980 ) and neurons have a significantly greater tendency to generate multiple population spikes than in the dorsal hippocampal formation (Gilbert et al, 1985). Other evidence from electrophysiological studies in both rats (Jung et al, 1994) and primates (Colombo et al, 1998) and from structural magnetic resonance imaging studies (Kjelstrup et al, 2002) furthermore suggest functional dissociation between the dorsal and ventral hippocampal formation.

While the dorsal hippocampal formation preferentially plays a role in spatial learning and memory (Bannerman et al, 2004), the ventral hippocampal subregion projects to the prefrontal cortex and is closely connected to bed nucleus of stria terminalis and the amygdala, as well as to other subcortical structures associated with the hypothalamic-pituitary-adrenal axis (Bannerman et al, 2004). Rat studies indicate that the ventral hippocampal formation is involved in anxiety (Alves et al, 2004) and that the ventral hippocampal formation and amygdala contribute distinctly to brain systems processing anxiety and/or fear (Mchugh et al, 2004). Since anxiety is present in the majority of patients with major depressive disorder (Fawcett and Kravitz, 1983; Fava et al, 2000), involvement of mainly the ventral, as opposed to the dorsal, hippocampal formation is likely to be important in the pathology of depression. 

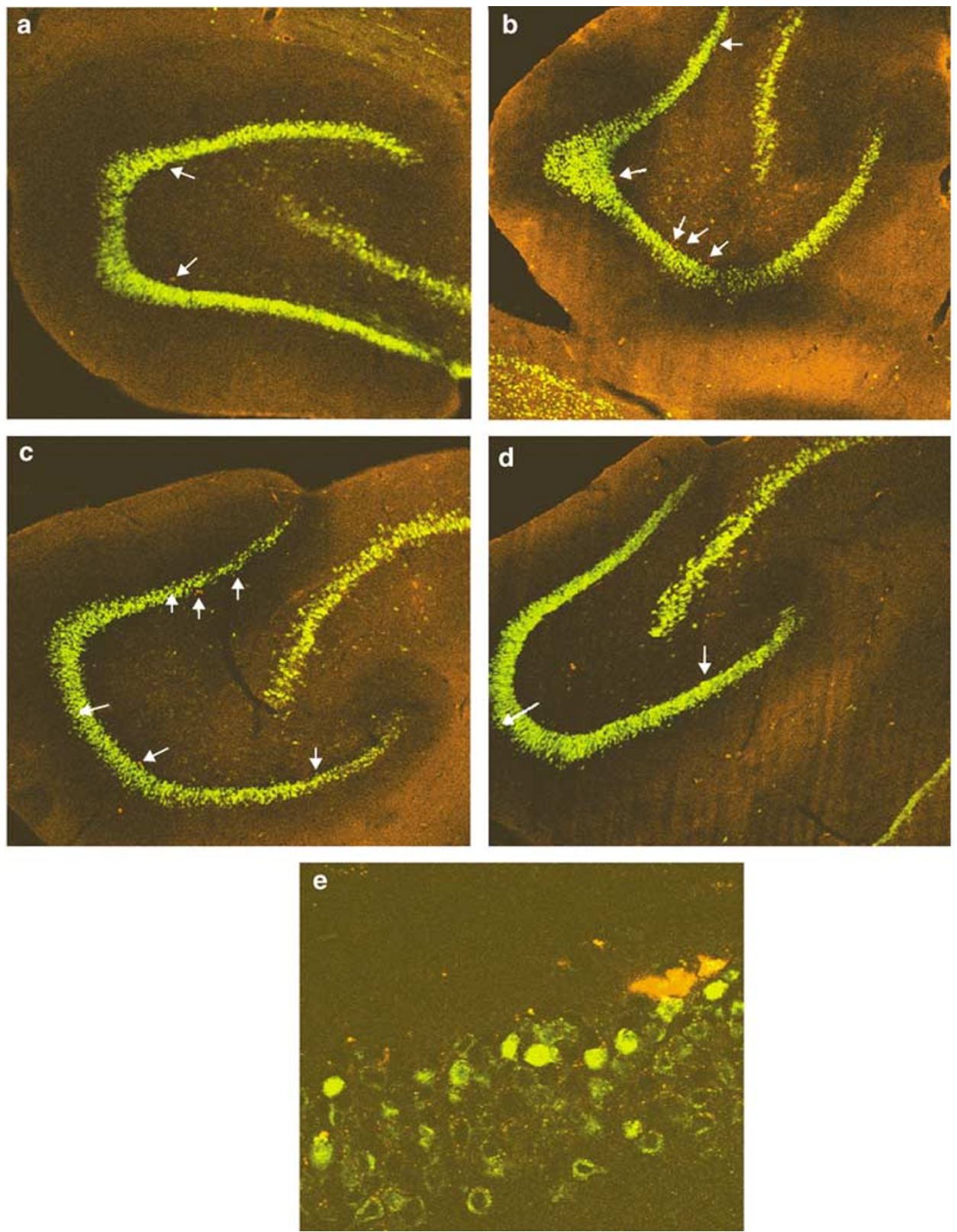

Figure 4 Cells clusters in granule cell layer (GCL) in the dentate gyrus of the ventral hippocampal formation. The number of BrdU-positive cells in GCL was reduced in the ventral subregion from animals exposed to CMS (a) compared with the unchallenged vehicle group (b). Chronic escitalopram treatment $(5 \mathrm{mg} / \mathrm{kg})$ reversed this effect in the responder group (c), but not in the nonresponder group (d). The cell clusters typically contain 3-12 cells (e) Magnification in (a-d) is 40000 and in (e) 100000 . BrdU-positive cells appearing in clusters are shown in red (indicated by arrows), mature neurons are shown in green.

From both a neuroanatomical and functional perspective we therefore find it interesting to look for local differences in cell proliferation within the hippocampal formation. To our knowledge all studies investigating adult neurogenesis in rodents so far have focused on the entire hippocampal formation as one unit or on the mediodorsal part of the formation.

In the present study, we found differences in the number of newly formed cells between the intrinsic subfields as well as between the dorsal and ventral part of the hippocampal formation when using the CMS model of depression. In this model, sucrose intake is considered a valid measure of sensitivity to reward and it is assumed, that CMS causes a generalized decrease in reward sensitivity (Muscat et al, 1991; Muscat and Willner, 1992; Willner et al, 1992, 1996; Willner, 1984, 1997, 2005).

After 2 weeks of administration of chronic mild stress the intake of sucrose solution was significantly diminished in the stressed animals compared to the unchallenged controls, indicating a stress-induced decrease in sensitivity to reward. Chronic treatment with escitalopram prevented the decrease in sucrose intake in approximately $50 \%$ of the treated rats (responders). The remaining animals did not respond to the treatment (nonresponders) (Figures 1 and 2).

In parallel studies rats were treated with either a double dose of escitalopram ( $10 \mathrm{mg} / \mathrm{kg} /$ day) or exposed to $5 \mathrm{mg} / \mathrm{kg}$ 


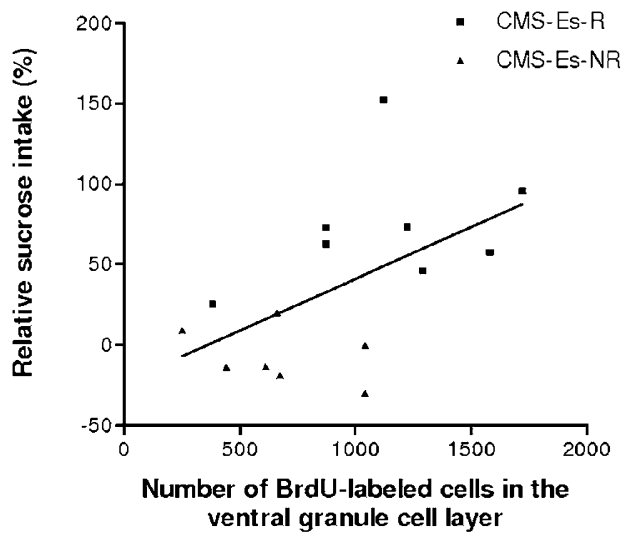

Figure 5 Sucrose intake vs number of new cells in the granule cell layer (GCL) of the ventral hippocampal formation. Sucrose intake is given as the relative increase in the average intake for all 4 weeks of escitalopram treatment compared to baseline. Sucrose intake shows a significantly positive correlation with the number of new cells $(p=0.036)$.

Table 2 Number of BrdU-Positive Cells in Hilus and Molecular Layer of Dentate Gyrus of Ventral Hippocampus

\section{Hilus \\ Molecular layer}

Animal group BrdU-labeled cells SEM BrdU-labeled cells SEM

\begin{tabular}{lllll}
\hline U-Es & 242 & 54 & 502 & 115 \\
U-V & 171 & 93 & $566 * * * *$ & 115 \\
CMS-V & 139 & 53 & 281 & 112 \\
CMS-Es-R & 216 & 53 & 434 & 112 \\
CMS-Es-NR & 165 & 56 & 380 & 119
\end{tabular}

U-Es, unchallenged escitalopram; U-V, unchallenged vehicle; CMS-V, stress vehicle; CMS-Es-R, stress escitalopram responders; CMS-Es-NR, stress

escitalopram nonresponders. No significant differences in cytogenesis in hilus were detected between the groups. In molecular layer (ML) the number of BrdU-positive cells was significantly reduced by CMS (CMS-V vs U-V). Significant differences were revealed in $M L$ when comparing the unchallenged vehicle group $(U-V)$ with the stress vehicle (CMS-V) and the nonresponder group (CMS-Es-NR).

$* *=p<0.01$ compared to stress vehicle.

$*=p<0.05$ compared to stress escitalopram nonresponders.

escitalopram twice a day. However, the ratio of responders to nonresponders remained the same (Table 1). Our data suggest that the observed treatment refraction is not caused by subdosing the antidepressant, but rather indicating that an unidentified pathological dose-independent mechanism is involved. The fraction of responders to escitalopram treatment in the CMS model closely mirrors the observed clinical efficacies (Einarson, 2004; Bondolfi et al, 1996).

The CMS model has been optimized and repeatedly run in our laboratory. The sucrose consumption test mirroring the decreased sensitivity to reward caused by CMS and the recovery from an anhedonic state provides highly reproducible results across different studies. In addition to escitalopram, another selective serotonin reuptake inhibitor, citalopram, and a serotonin/norepinephrine reuptake inhibitor, duloxetine, were tested (data not shown). In our

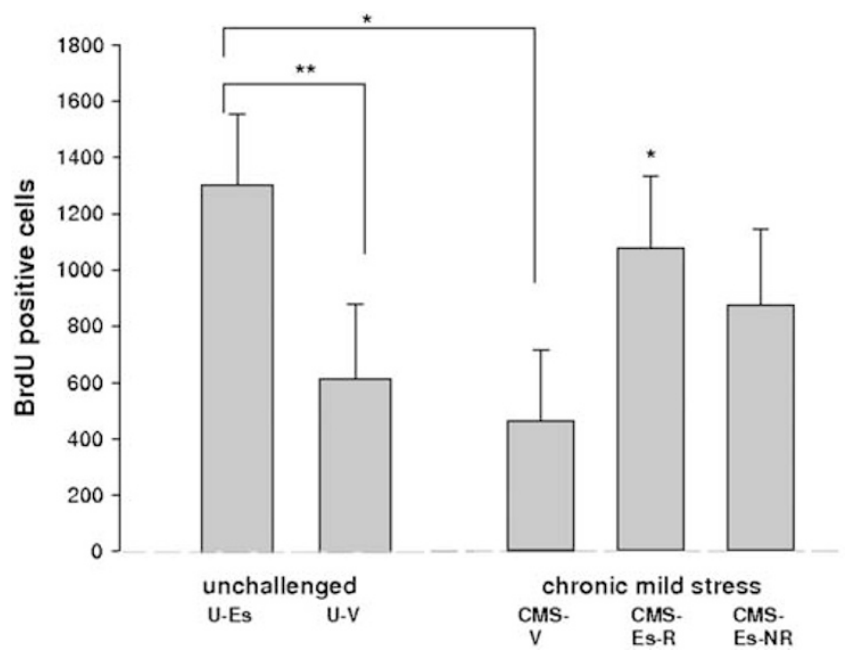

Figure 6 Total number of BrdU-positive cells in the granule cell layer (GCL) of the dorsal hippocampal formation. Effect of chronic mild stress on cell proliferation in the dorsal hippocampal formation was insignificant (CMS-V vs U-V). A significant increase in cell proliferation after escitalopram treatment $(5 \mathrm{mg} / \mathrm{kg}$ ) was observed in the unchallenged escitalopram group (U-Es compared to U-V). The number of new cells was significantly higher in the unchallenged escitalopram group (U-Es) compared with stress vehicle group (CMS-V). The mean number of BrdU-labeled cells was also higher in CMS escitalopram groups (CMS-Es-R and CMS-Es-NR) compared with the CMS vehicle group (CMS-V), but the increase was only significant for the responder group (CMS-Es-R). However, the number of new cells did not differ significantly between the responder (CMS-Es-R) and nonresponder (CMS-Es-NR) groups. Data are presented as means \pm SEM and were analyzed with analysis of variance (ANOVA) and LSD post hoc test. $*=p<0.05$, $* *=p<0.01$ compared with stress vehicle group or as indicated.

hands the drug-exposed population reproducibly splits into a responder and nonresponder group, and the percentage of animals in each group was highly comparable between different drug treatment regimens.

We therefore suggest that in addition to modeling anhedonia and delay in antidepressant response, CMS is also a valid model of therapy resistance.

In the present study, we used the CMS model to investigate the changes in cell proliferation rates in the dentate gyrus of the hippocampal formation of anhedonic rats compared with rats that recovered from anhedonia after chronic escitalopram treatment.

We investigated the first stage in neurogenesis, that is, cell proliferation, and found different profiles between the different CMS groups in SGZ of the dentate gyrus. Most of the precursor cells from SGZ divide and if they survive they will generate new neurons (Seri et al, 2001). This allows us to speculate that our data indicate that stress and/or escitalopram treatment affect not only the generation of new cells, but also new neurons, in the (GCL) in the dentate gyrus of the CMS animals. Results from neurogenesis studies in other animal models of depression support our speculations.

In the tree shrew the decrease in neurogenesis and overall hippocampal volumen by psychological stress was reversed by chronic treatment with tianeptine or clomipramine (Czeh et al, 2001; van der Hart et al, 2002). Chronic fluoxetine treatment reversed a decrease in neurogenesis in 


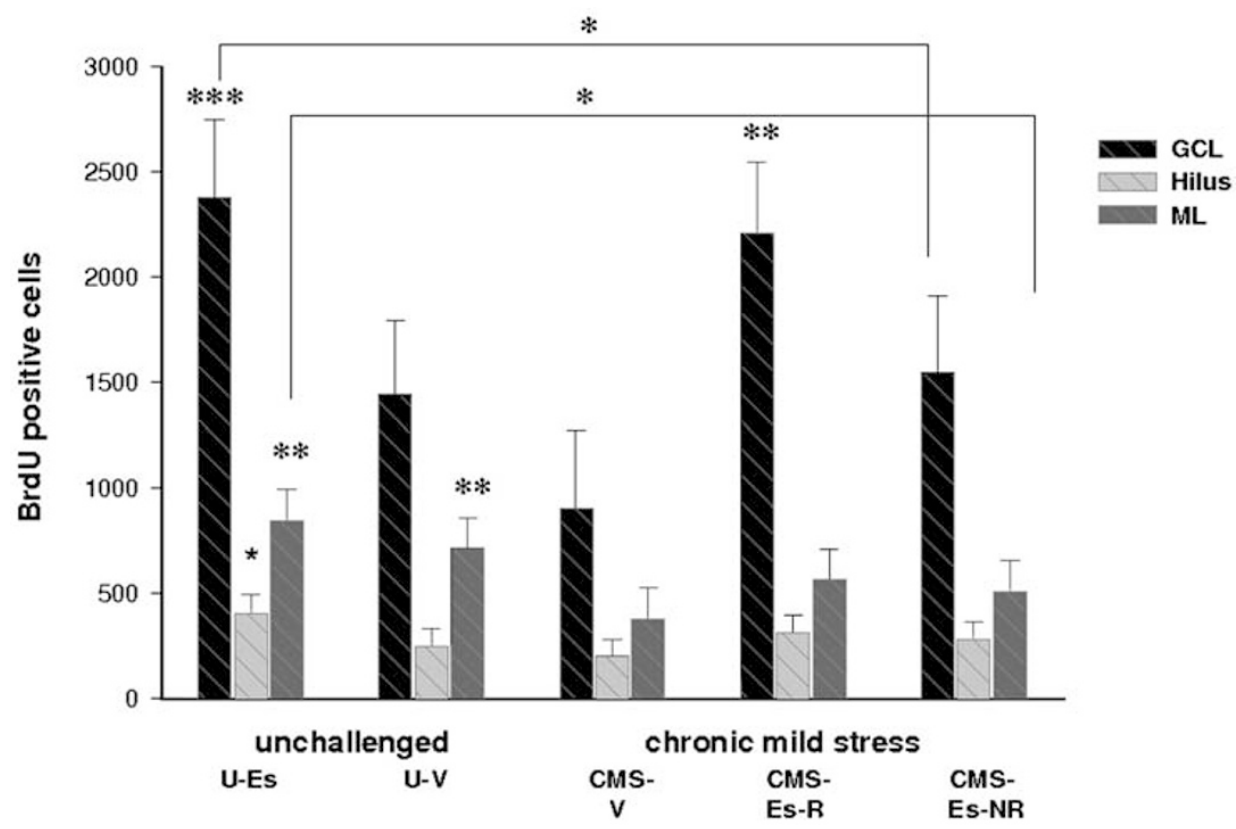

Figure 7 Distribution of BrdU-positive cells in the dentate gyrus through the entire hippocampal formation. A significant inhibition of cell proliferation by stress was observed in the molecular layer (ML) (CMS-V compared with U-V). The number of BrdU-positive cells in GCL was significantly higher in responder (CMS-Es-R) compared with stress vehicle group (CMS-V). A significantly higher number of BrdU-positive cells in GCL, hilus and ML was found in the unchallenged escitalopram group (U-Es) compared with the stress vehicle (CMS-V) group. Data are presented as means \pm SEM and were analyzed with analysis of variance (ANOVA) and LSD post hoc test. $*=p<0.05$, $* *=p<0.01$, and $* * * *=p<0.001$ compared to stress vehicle group or as indicated.

the (GCL) of the hippocampal formation in the learned helplessness model for depression and in the maternal separation model (Malberg and Duman, 2003; Lee et al, 2001). Our data on escitalopram are in agreement with these findings.

For unchallenged animals the phenomenon of antidepressive medications increasing neurogenesis in GCL of the hippocampal formation is well established. The antidepressants shown to increase cell proliferation in unchallenged animals are: fluoxetine, reboxetine, tranylcypromine, imipramine (Malberg et al, 2000; Santarelli et al, 2003). For the first time we show that also escitalopram increases cell proliferation in the hippocampal formation of unchallenged animals and that this action is selective for the dorsal part of the hippocampal formation. We report different actions of escitalopram on hippocampal cell proliferation in unchallenged and anhedonic animals. In the animals recovering from anhedonia, escitalopram increases cell proliferation in GCL in both the dorsal and ventral part of the hippocampal formation, whereas this action is limited to the dorsal region in unchallenged animals.

Previously, an association between impaired adult neurogenesis and changes in behavior was investigated in mice (Santarelli et al, 2003). In the novelty-suppressed feeding (NSF) test an irradiation impaired hippocampal neurogenesis was shown to correlate with long latency of feeding, and this effect was not reversed by chronic treatment with antidepressants. In contrast to sham mice, antidepressant treatment did not reverse the bad coat state and the grooming latency in the grooming test in irradiated stress exposed animals. While NSF, coat state assessment and grooming are associated with anxiety and stress, the present study correlates an anhedonia-like behavior with cyto- genesis in the hippocampal formation (Figure 5). Thus we are capable of addressing a major symptom of depression in the CMS model, which we find to be of major importance. A possible interpretation of our data is that stress-induced anhedonia-like behavior in rats may be caused by a diminished cytogenesis in GCL of the ventral hippocampal formation (Figure 3). However, it should be stated that our data are only correlative. It cannot be excluded that other cellular or neurochemical alterations are accounting for changes in sucrose intake. The second finding is that chronic escitalopram treatment can reverse both of these effects but only in approximately $50 \%$ of the treated population. We find an overall positive correlation between recovery from behavioral deficits and cell proliferation rates in the ventral hippocampal formation (Figure 5). On the basis of these results we hypothesize that the treatment resistance phenomenon involves pathological inhibition of antidepressant actions on cell proliferation in the ventral hippocampal formation. Finally, our study demonstrates that the actions of antidepressant drugs differ drastically when comparing anhedonic and unchallenged animals (Figures 3 and 6). These facts emphasize the importance of using animal models when investigating the pathology of depression and the mechanisms of antidepressant therapy. Furthermore, our data highlight the need of segregating the animals on the basis of their behavior into responder and nonresponder groups in order to reveal mechanisms underlying therapeutic drug actions.

On the basis of the anatomical and functional differences we hypothesize that dorsal and ventral regions of the hippocampal formation play different roles in the pathology of depressive disorders and that restoration of cell proliferation rates in the ventral hippocampal formation is 
a key step in obtaining recovery. In order to improve clinical drug efficacy we therefore suggest that future treatment strategies are aimed at achieving a stimulation of hippocampal cytogenesis with specific attention to the ventral subregion.

\section{ACKNOWLEDGEMENTS}

We thank JM Kuhn for help with the statistical analysis and J Hellsten, MJ West, C Sanchéz, A Mørk, and R Rosenberg for comments and suggestions. This work was supported by the Danish Research Agency and the Lundbeck Foundation.

\section{REFERENCES}

Alonso R, Griebel G, Pavone G, Stemmelin J, Le Fur G, Soubrie P (2004). Blockade of CRF1 or V-1b receptors reverses stressinduced suppression of neurogenesis in a mouse model of depression. Mol Psychiatr 9: 278-286.

Alves SH, Pinheiro G, Motta V, Landeira-Fernandez J, Cruz APM (2004). Anxiogenic effects in the rat elevated plus-maze of 5-HT2C agonists into ventral but not dorsal hippocampus. Behav Pharmacol 15: 37-43.

Bannerman DM, Rawlins JNP, Mchugh SB, Deacon RMJ, Yee BK, Bast $\mathrm{T}$ et al (2004). Regional dissociations within the hippocampus-memory and anxiety. Neurosci Biobehav Rev 28: 273-283.

Bondolfi G, Chautems C, Rochat B, Bertschy G, Baumann P (1996). Non response to citalopram in depressive patients: pharmacokinetic and clinical consequences of a fluvoxamine augmentation. Psychopharmacology 128: 421-425.

Bremner JD, Narayan M, Anderson ER, Staib LH, Miller HL, Charney DS (2000). Hippocampal volume reduction in major depression. Am J Psychiatr 157: 115-117.

Brown ES, Rush AJ, McEwen BS (1999). Hippocampal remodeling and damage by corticosteroids: implications for mood disorders. Neuropsychopharmacology 21: 474-484.

Colombo M, Fernandez T, Nakamura K, Gross CG (1998). Functional differentiation along the anterior-posterior axis of the hippocampus in monkeys. J Neurophysiol 80: 1002-1005.

Czeh B, Michaelis T, Watanabe T, Frahm J, de Biurrun G, van Kampen $M$ et al (2001). Stress-induced changes in cerebral metabolites, hippocampal volume, and cell proliferation are prevented by antidepressant treatment with tianeptine. Proc Natl Acad Sci USA 98: 12796-12801.

Einarson TR (2004). Evidence based review of escitalopram in treating major depressive disorder in primary care. Int Clin Psychopharmacol 19: 305-310.

Fava M, Rankin MA, Wright EC, Alpert JE, Nierenberg AA, Pava J et al (2000). Anxiety disorders in major depression. Comprehensive Psychiatr 41: 97-102.

Fawcett J, Kravitz HM (1983). Anxiety syndromes and their relationship to depressive-illness. J Clin Psychiatr 44: 8-11.

Feng ZD, McCulloch CE (1996). Using bootstrap likelihood ratios in finite mixture models. J $R$ Statist Soc Ser B-Methodological 58: 609-617.

Fuchs E, Czeh B, Flugge G (2004). Examining novel concepts of the pathophysiology of depression in the chronic psychosocial stress paradigm in tree shrews. Behav Pharmacol 15: 315-325.

Gage FH, Thompson RG (1980). Differential distribution of norepinephrine and serotonin along the dorsal-ventral axis of the hippocampal-formation. Brain Res Bull 5: 771-773.

Gilbert M, Racine RJ, Smith GK (1985). Epileptiform burst responses in ventral $v s$ dorsal hippocampal slices. Brain Res 361: 389-391.
Gould E, McEwen BS, Tanapat P, Galea LAM, Fuchs E (1997). Neurogenesis in the dentate gyrus of the adult tree shrew is regulated by psychosocial stress and NMDA receptor activation. J Neurosci 17: 2492-2498.

Gould E, Tanapat P, McEwen BS, Flugge G, Fuchs E (1998). Proliferation of granule cell precursors in the dentate gyrus of adult monkeys is diminished by stress. Proc Nat Acad Sci USA 95: 3168-3171.

Jung MW, Wiener SI, Mcnaughton BL (1994). Comparison of spatial firing characteristics of units in dorsal and ventral hippocampus of the rat. J Neurosci 14: 7347-7356.

Kjelstrup KG, Tuvnes FA, Steffenach HA, Murison R, Moser EI, Moser MB (2002). Reduced fear expression after lesions of the ventral hippocampus. Proc Natl Acad Sci USA 99: 10825-10830.

Lee HJ, Kim JW, Yim SV, Kim MJ, Kim SA, Kim YJ et al (2001). Fluoxetine enhances cell proliferation and prevents apoptosis in dentate gyrus of maternally separated rats. Mol Psychiatr 6: $725-728$.

Madsen TM, Treschow A, Bengzon J, Bolwig TG, Lindvall $\mathrm{O}$, Tingstrom A (2000). Increased neurogenesis in a model of electroconvulsive therapy. Biol Psychiatr 47: 1043-1049.

Magarinos AM, McEwen BS, Flugge G, Fuchs E (1996). Chronic psychosocial stress causes apical dendritic atrophy of hippocampal CA3 pyramidal neurons in subordinate tree shrews. J Neurosci 16: 3534-3540.

Malberg JE, Duman RS (2003). Cell proliferation in adult hippocampus is decreased by inescapable stress: reversal by fluoxetine treatment. Neuropsychopharmacology 28: 1562-1571.

Malberg JE, Eisch AJ, Nestler EJ, Duman RS (2000). Chronic antidepressant treatment increases neurogenesis in adult rat hippocampus. J Neurosci 20: 9104-9110.

Malberg JE, Schechter LE (2005). Increasing hippocampal neurogenesis: a novel mechanism for antidepressant drugs. Curr Pharmaceut Des 11: 145-155.

Mchugh SB, Deacon RMJ, Rawlins JNP, Bannerman DM (2004). Amygdala and ventral hippocampus contribute differentially to mechanisms of fear and anxiety. Behav Neurosci 118: 63-78.

McLachlan GJ, Bean RW, Peel D (2002). A mixture model-based approach to the clustering of microarray expression data. Bioinformatics 18: 413-422.

Montgomery SA, Loft H, Sanchez C, Reines EH, Papp M (2001). Escitalopram (S-enantiomer of citalopram): clinical efficacy and onset of action predicted from a rat model. Pharmacol Toxicol 88: $282-286$.

Moreau JL, Jenck F, Martin JR, Mortas P, Haefely W (1993). Effects of moclobemide, a new generation reversible mao-A inhibitor, in a novel animal-model of depression. Pharmacopsychiatry 26: 30-33.

Moreau JL, Scherschlicht R, Jenck F, Martin JR (1995). Chronic mild stress-induced anhedonia model of depression; sleep abnormalities and curative effects of electroshock treatment. Behav Pharmacol 6: 682-687.

Moser MB, Moser EI (1998). Functional differentiation in the hippocampus. Hippocampus 8: 608-619.

Muscat R, Kyprianou T, Osman M, Phillips G, Willner P (1991). Sweetness-dependent facilitation of sucrose drinking by raclopride is unrelated to calorie content. Pharmacol Biochem Behav 40: 209-213.

Muscat R, Willner P (1992). Suppression of sucrose drinking by chronic mild unpredictable stress: a methodological analysis. Neurosci Biobehav Rev 16: 507-517.

Papp M, Moryl E, Willner P (1996). Pharmacological validation of the chronic mild stress model of depression. Eur J Pharmacol 296: $129-136$.

Papp M, Willner P, Muscat R (1991). An animal model of anhedonia: attenuation of sucrose consumption and place preference conditioning by chronic unpredictable mild stress. Psychopharmacology (Berlin) 104: 255-259. 
Paxinos G, Watson C (1986). The Rat Brain in Stereotactic Coordinates. Academic Press: Sydney, Australia.

Sanchez C, Gruca P, Papp M (2003). R-citalopram counteracts the antidepressant-like effect of escitalopram in a rat chronic mild stress model. Behav Pharmacol 14: 465-470.

Santarelli L, Saxe M, Gross C, Surget A, Battaglia F, Dulawa S et al (2003). Requirement of hippocampal neurogenesis for the behavioral effects of antidepressants. Science 301: 805-809.

Schildkraut JJ (1965). The catecholamine hypothesis of affective disorders: a review of supporting evidence. J Neuropsychiatr Clin Neurosci 7: 524-533.

Seri B, Garcia-Verdugo JM, McEwen BS, Alvarez-Buylla A (2001). Astrocytes give rise to new neurons in the adult mammalian hippocampus. J Neurosci 21: 7153-7160.

Sheline YI, Gado MH, Kraemer HC (2003). Untreated depression and hippocampal volume loss. Am J Psychiatr 160: 1516-1518.

Tanapat P, Galea LAM, Gould E (1998). Stress inhibits the proliferation of granule cell precursors in the developing dentate gyrus. Int J Dev Neurosci 16: 235-239.

Vaidya VA, Duman RS (2001). Depression-emerging insights from neurobiology. Br Med Bull 57: 61-79. van der Hart MGC, Czeh B, de Biurrun G, Michaelis T, Watanabe $\mathrm{T}$, Natt $\mathrm{O}$ et al (2002). Substance $\mathrm{P}$ receptor antagonist and clomipramine prevent stress-induced alterations in cerebral metabolites, cytogenesis in the dentate gyrus and hippocampal volume. Mol Psychiatr 7: 933-941.

Willner P (1984). The validity of animal models of depression. Psychopharmacology (Berlin) 83: 1-16.

Willner P (1997). Validity, reliability and utility of the chronic mild stress model of depression: a 10-year review and evaluation. Psychopharmacology (Berlin) 134: 319-329.

Willner P (2005). Chronic mild stress (CMS) revisited: consistency and behavioural-neurobiological concordance in the effects of CMS. Neuropsychobiology 52: 90-110.

Willner P, Moreau JL, Nielsen CK, Papp M, Sluzewska A (1996). Decreased hedonic responsiveness following chronic mild stress is not secondary to loss of body weight. Physiol Behav 60: 129-134.

Willner P, Muscat R, Papp M (1992). Chronic mild stress-induced anhedonia: a realistic animal model of depression. Neurosci Biobehav Rev 16: 525-534.

Willner P, Towell A, Sampson D, Sophokleous S, Muscat R (1987). Reduction of sucrose preference by chronic unpredictable mild stress, and its restoration by a tricyclic antidepressant. Psychopharmacology (Berlin) 93: 358-364. 